\title{
Convenient but powerful method to dope single-walled carbon nanotube films with iodonium salts
}

\author{
B. Kumanek ${ }^{1}$ (1) Ł. Przypis ${ }^{1} \cdot$ P. S. Wróbel ${ }^{2} \cdot$ M. Krzywiecki ${ }^{3} \cdot$ K. Z. Walczak ${ }^{1} \cdot$ D. Janas ${ }^{1}$
}

Received: 28 May 2019 / Accepted: 9 August 2019 / Published online: 21 August 2019

(C) The Author(s) 2019

\begin{abstract}
Single-walled carbon nanotubes (SWCNTs) demonstrate unique properties of both electrical and thermal conductivity, but at the moment of producing a network composed of multiple nanotubes, these values sharply decrease. It is associated with the presence of defects in the nanotube network structure, as well as with the dissipation phenomena at the junctions between SWCNTs. One of the methods to alleviate this problem is doping. In our study, we obtained a film made of high-quality SWCNTs doped with three types of iodonium salts: Barluenga's reagent (bispyridineiodonium tetrafluoroborate, $\mathrm{IPy}_{2} \mathrm{BF}_{4}$ ), pyridine iodine monochloride (IPyCl) and diphenyliodonium chloride (DPIC). We recorded a significant improvement in electrical properties after doping with $\mathrm{IPy}_{2} \mathrm{BF}_{4}$ and $\mathrm{IPyCl}$, by as much as $224 \%$ and $322 \%$, respectively. What is more, we noted improvement in thermal conductivity, which amounted to over $50 \%$ when the material was doped with IPyCl. Our research indicates that permanent doping of CNT-based ensembles is possible with iodonium salts. The process significantly improves electrical conductivity, and the compounds themselves are more convenient to work with rather than when other halogen-based dopants are used commonly for this purpose.
\end{abstract}

Keywords Carbon nanotubes $\cdot$ Doping $\cdot$ Electrical properties $\cdot$ Thermal properties $\cdot$ Iodonium salts

\section{Introduction}

Carbon nanotubes (CNTs), since the moment of discovery (Iijima 1991), have enjoyed the attention of scientists, because they demonstrate unique mechanical (Gonnet et al. 2006), thermal (Berber et al. 2000; Fujii et al. 2005; Kumanek and Janas 2019) and electrical (Chen et al. 2008; Brady et al. 2016; Friedman et al. 2017) properties. They

Electronic supplementary material The online version of this article (https://doi.org/10.1007/s13204-019-01133-y) contains supplementary material, which is available to authorized users.

B. Kumanek

bogumila.kumanek@polsl.pl

D. Janas

dawid.janas@polsl.pl

1 Department of Chemistry, Silesian University of Technology, B. Krzywoustego 4, 44-100 Gliwice, Poland

2 Centre of Polymer and Carbon Materials Polish Academy of Science, M. Curie-Sklodowskiej 34, 41-819 Zabrze, Poland

3 Institute of Physics-CSE, Silesian University of Technology, Konarskiego 22B, 44-100 Gliwice, Poland may have metallic and semiconductor properties (McEuen et al. 2002) depending on the particular way the carbon atoms are arranged at the nanoscale. This means that the geometry of nanotubes, in particular their internal structure, affects their thermal and electrical conductivity. Individual CNTs are characterized by the carrier mobility of up to $10,000 \mathrm{~cm}^{2} / \mathrm{V} \mathrm{s}$ at $50 \mathrm{~K}$ (Zhou et al. 2005) which is much higher than in the case of silicon, and the electrical current density of up to $4 \times 10^{9} \mathrm{~A} / \mathrm{cm}^{2}$ (Javey et al. 2004), which is better than the properties of a typical metal such as copper or aluminum by as much as three orders of magnitude (Hong and Myung 2007). Due to their properties, CNTs are applied as very sensitive electrochemical sensors (Gao et al. 2012), biosensors (Wang 2005), batteries (Landi et al. 2009), energy devices (Blackburn et al. 2018), transparent conductors (Wu et al. 2004) and others (Blackburn et al. 2018).

All the time, there is a growing demand for semiconductors or conductors with improved electrical properties, and so, given their characteristics, it is not surprising that nanocarbon materials are envisioned as one of the most promising candidates on this front. What is even more beneficial is that rich chemistry of carbon enables one to tweak their electrical properties with ease. One of the simplest methods 
of doing so is introduction of a dopant in onto the surface of a CNT or into the structure. The dopant affects the distribution of the electron charge of the nanotube when it is physically or chemically bonded with it, which directly affects its Fermi level. Doping may be conducted using encapsulation (Fukumaru et al. 2015), via modification of crystal structure of carbon nanotubes by heteroatoms (Liu et al. 2016b) or by exposure of the surface of carbon nanotubes to the doping agents (Janas et al. 2013b, 2014; Tonkikh et al. 2015; Zhao et al. 2011; Lee et al. 1997). The injected heteroatom or compound could be classified as an electron-donor (n-type) or electron-acceptor (p-type). In the literature, halogens (Janas et al. 2014; Tonkikh et al. 2015; Zhao et al. 2011), interhalogens (Janas et al. 2017a) or strong acids (Janas et al. 2013b; Lee et al. 1997) are mainly used as p-type dopants and nitrogen compounds (Janas 2018) or alkali metals (Lee et al. 1997) as n-type dopants. To this day, one of the highest gain in electrical conductivity was obtained by doping carbon nanotubes with halogens to create cables exceeding specific electrical conductivity of metals (Zhao et al. 2011).

Modifications of CNT ensembles using halogen compounds may be conducted in different ways. One of the simple techniques involves exposing CNTs to chlorine, bromine or iodine vapours (Janas et al. 2014; Qiu et al. 2018). Another method is immersing CNTs in a solution containing halogen compounds such as: $\mathrm{SOCl}_{2}$ (Dettlaff-Weglikowska et al. 2005), $I_{2}$ (Janas et al. 2014), $\mathrm{PBr}_{3}$ (Fanchini et al. 2007) or ICl (Janas et al. 2014). Finally, it can be realized by Hunsdiecker reaction with oxidized CNTs (Janas et al. 2014), which results in favorable chemical modification. These processes suffer many disadvantages, such as the requirement of large excess of halogen-bearing reagent, the necessity to employ harsh reaction conditions (which demands appropriate health and safety measures) or long processing times. Furthermore, handling gaseous halogens is problematic because these compounds are toxic, corrosive and demand challenging conditions to introduce these atoms into the structure of CNTs. Despite this restriction, halogen doping is an effective way of improving electrical conductivity of CNT films and iodine compounds play a special role in this field because of their strong action on the electrical properties of nanocarbon materials (Janas et al. 2014; Zhan et al. 2015). Therefore, the search for mild approaches how to bring iodine and carbon nanomaterials together is of great interest.

Recently, new reports about effective, mild and safe iodination procedures emerged for arenes (Espuña et al. 2004; Barluenga et al. 2007; Racys et al. 2016) and heterocycles (Barluenga et al. 1993; Hamri et al. 2012; Przypis and Walczak 2019). In this subject, hypervalent iodine reagents have been at the forefront of synthetic methodology developments in the past 20 years (Merritt and Olofsson 2009; Yoshimura and Zhdankin 2016). Multiple examples of iodonium salt usage in organic synthesis have been reported in recent literature (Barluenga et al. 1993; Espuña et al. 2004; Hamri et al. 2012; Borzenko et al. 2015; Racys et al. 2016; Iskra and Murphree 2017; Liu et al. 2017; Przypis and Walczak 2019). Aryliodonium and diaryliodonium salts play a special role in this field because they are air- and moisture stable and soluble in many organic solvents despite their reactive framework. The idea to use iodonium salt in the nanocarbon realm was recently explored by He and Swager (2016), who employed them for introduction of the aryl moiety into their structure mediated by sodium naphthalide. Herein, we present another facet of iodonium salts, which we successfully employed for modification of CNT electronic properties by iodine under mild conditions.

The research described by us constitutes an alternative pathway to the previously taken routes of halogen introduction into the CNT network and solve the drawbacks described above. For this purpose, we designed and synthesized three different iodonium salts: Barluenga's reagent (bispyridineiodonium tetrafluoroborate, $\mathrm{IPy}_{2} \mathrm{BF}_{4}$ ), pyridine iodine monochloride (IPyCl) and diphenyliodonium chloride (DPIC) of various electronic and structural characteristics. Tailor-made iodine-bearing compounds were very successful in doping CNT films as compared with iodine monochloride, which we used as reference. Because of their high stability in the ambient and elimination of the common problem of desorption, evaporation or sublimation of halogen species from the doped material, these compounds appear as very attractive agents to tailor the electronic properties of nanocarbon materials.

\section{Experimental}

\section{Doping of SWCNT films with iodonium salts}

Thin free-standing CNTs films were obtained using a method reported previously by us (Janas et al. 2017b) from high-quality single-walled carbon nanotubes (SWCNTs; Tuball $^{\mathrm{TM}}$ ) purchased from OCSiAl. In brief, this method involved dispersing nanocarbon material in an acetone/ toluene mixture assisted by the presence of ethyl cellulose (EC). After sonication, during which the mixture reached uniform dispersion, it was gradually placed in the form of several layers on Kapton foil. After evaporation of the whole solvent, the dry film, which formed on the substrate, was delicately peeled off. Then, the CNT film was cleaned of EC by rapid thermal annealing (Janas and Stando 2017). The obtained CNT film was immersed in 0.1-M solution of selected iodonium salt in $\mathrm{CH}_{2} \mathrm{Cl}_{2}$ for $60 \mathrm{~s}$, and then placed in a vacuum desiccator to evaporate the solvent. 
Fig. 1 Structure of iodonium salts: diphenyliodonium chloride (DPIC), bispyridine iodonium tetrafluoroborate $\left(\mathrm{IPy}_{2} \mathrm{BF}_{4}\right)$ and pyridineiodonium chloride (IPyCl)

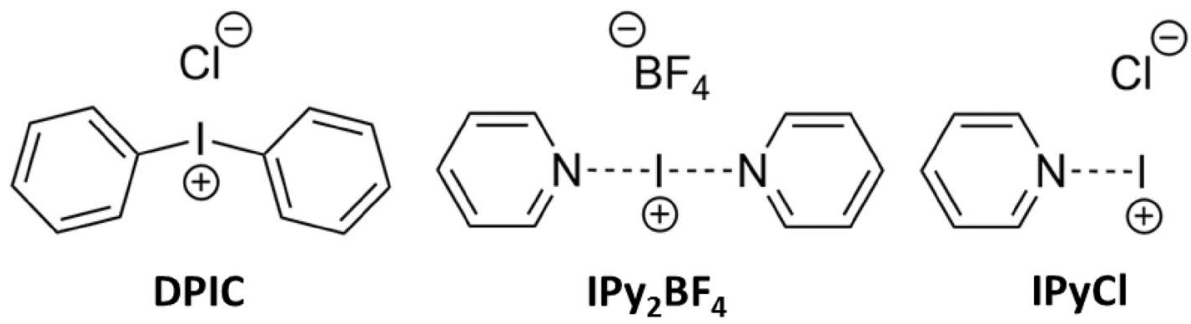

Information about the method of synthesis of iodonium salts can be found in the included Supplementary Information file. Figure 1 depicts the structure of the doping agents obtained for the study.

\section{Characterization of CNT films}

To fully characterize the obtained pristine and doped SWCNTs films, the following methods were employed.

Raman spectroscopy (Alpha 300 Witec, Germany) with a confocal microscope and an Nd-YAG laser (532 nm, max. power $23 \mathrm{~mW}$, power of measurements $2.3 \mathrm{~mW}$ ) were used to analyze the surface of the pristine and doped CNTs. The measurement was conducted with static mode centered at $1500 \mathrm{~cm}^{-1}$ (which corresponds to acquisition window from 0 to $3500 \mathrm{~cm}^{-1}$ ) with 25 accumulations and integration time of $10 \mathrm{~s}$ to obtain a signal essentially without noise. The intensity ratio $I_{\mathrm{D}} / I_{\mathrm{G}}$, which informs us about the degree of structural perfection of the material, was calculated.

Scanning electron microscopy (FEI Quanta 250 FEG) was used to characterize morphologies of pristine CNTs and CNTs after doping. The micrographs of samples were taken in high vacuum using field emission gun operated at $15 \mathrm{kV}$.

Thermogravimetric analysis (TGA2 Mettler Toledo) was performed from 25 to $1000^{\circ} \mathrm{C}$, with heating rate $10{ }^{\circ} \mathrm{C} / \mathrm{min}$ and in the stream of air of $30 \mathrm{ml} / \mathrm{min}$.

X-ray photoemission and Auger spectra were obtained using PREVAC EA15 hemispherical electron Energy analyzer equipped with X-ray source (PREVAC dual-anode XR40B, Al-Ka line, energy $1486.60 \mathrm{eV}$ ) and 2D-MCP detector. The measurements were carried out using the following conditions: $0.9 \mathrm{eV}$ scanning step, $200 \mathrm{eV}$ pass energy and $2 \times 10^{-8} \mathrm{~Pa}$ system base pressure. The binding energy (BE) scale was calibrated by positioning the reference peaks of $\mathrm{Au}$ $4 f_{7 / 2}(84.0 \mathrm{eV})$. The data recorded were fitted using CASA XPS embedded algorithms as well as relative sensitivity factors. Background modelled by the Shirley function was subtracted. It was determined that the estimated uncertainty for the energy position of components amounted to $0.1 \mathrm{eV}$.

$\mathrm{X}$-ray powder diffraction (XRD) was used to detect impact of iodonium salts on the crystallinity of CNTs. The analysis was obtained using an X-ray diffractometer Empyrean (PANAlytical equipped with a PIXcel ${ }^{3 \mathrm{D}}$ detector). The measurements were taken using Bragg-Brentano configuration. Conditions of the measurement: $\mathrm{Cu} \mathrm{K} \alpha$ $\lambda=1.5406 \AA, 40 \mathrm{kV}, 30 \mathrm{~mA}$.

The electrical conductivity measurement was made with a 4-point method using a Keithley 2450 SourceMeter. A CNT cut film with dimensions of $3 \mathrm{~mm} \times 60 \mathrm{~mm}$ was placed, using silver paste, on the copper electrodes to eliminate the problem of contact resistance. The whole measurement set-up was placed on an insulating glass substrate (Fig. 2). The value of electrical resistivity under electric current of $100 \mathrm{~mA}$ was measured, and then the value of electrical conductivity was calculated by taking into the account the dimensions of the samples obtained from optical microscope. At least five measurements were made for each sample. The results were averaged and statistical error was calculated.

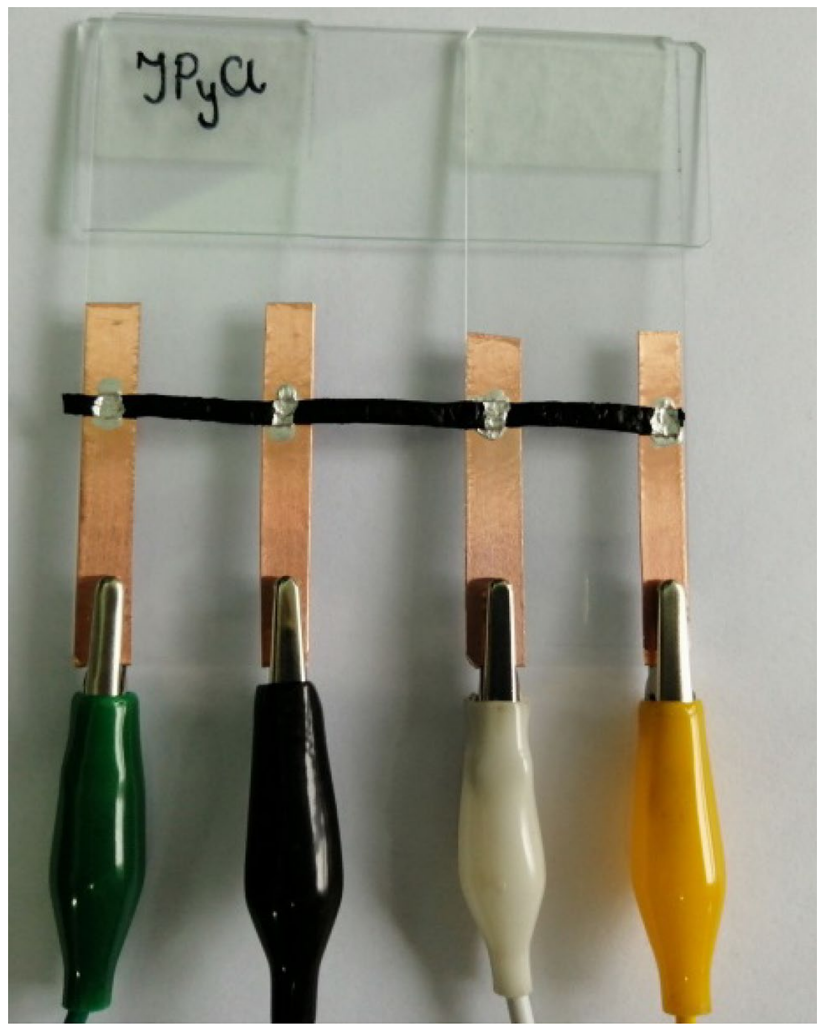

Fig. 2 Set-up with a sample for measurement of electrical conductivity 
Thermal conductivity was measured using steady-state method with infrared thermography (Wang et al. 2008; Zhang et al. 2012; Liu et al. 2016a). The measurement consisted in recording the temperature profile along the whole length of the sample, while direct current of specific parameters flows through it. The temperature of the sample as a function of its length was recorded using an IR thermometer (FLIR ETS 320) and modeled using the following equation:

$\kappa=\frac{U \times I \times 0.5 L}{4 \times w \times d \times\left(T_{\mathrm{m}}-T_{0}\right)}$,

where $\kappa$ is thermal conductivity, $U$ is voltage, $l$ is current, $L, w$ and $t$ are length, width and thickness of the sample, respectively. $T_{\mathrm{o}}$ is temperature on ends of the sample and

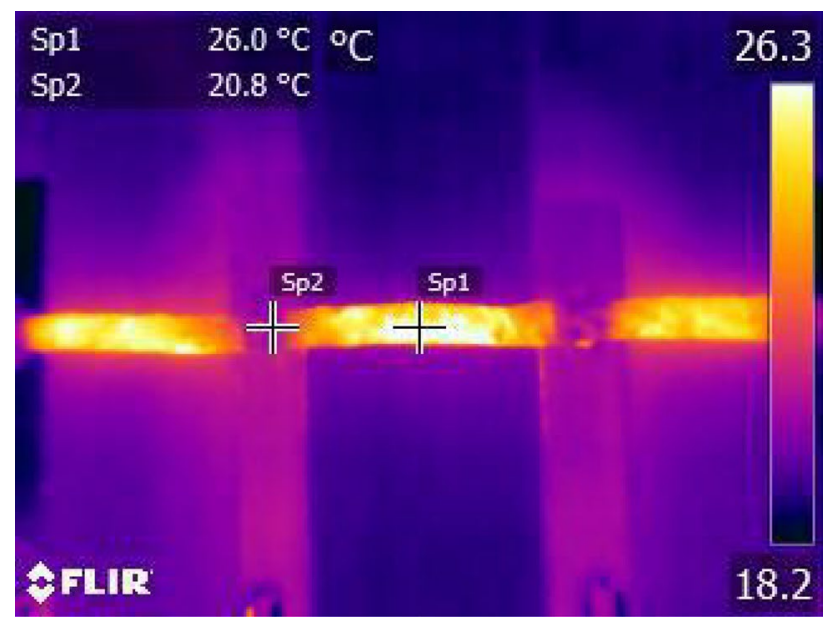

Fig. 3 Infrared thermograph of the measured samples
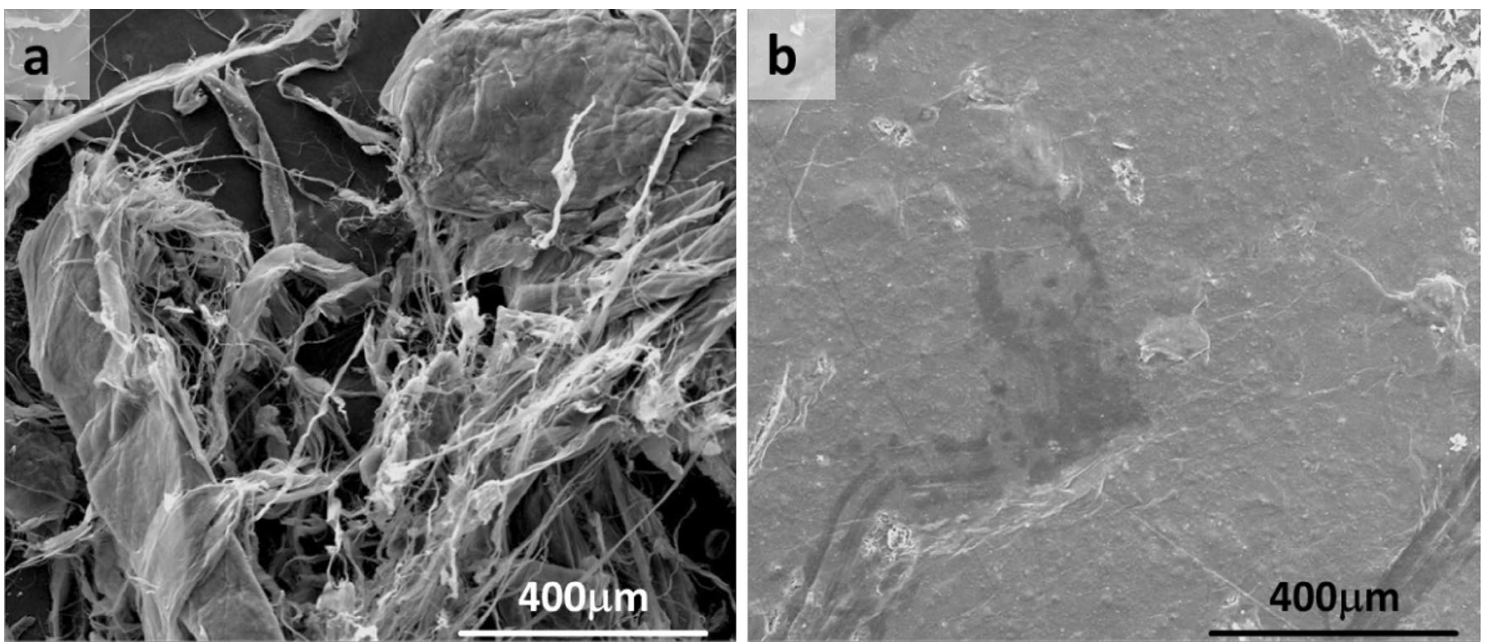

Fig. 4 SEM micrographs of a parent CNT powder and $\mathbf{b}$ freestanding film made from it at $\times 250$ magnification

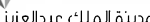

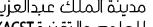

Springer 
Fig. 5 SEM micrographs of films from a pristine CNT and doped by iodine compounds, b CNT-DPIC, c CNT-IPy ${ }_{2} \mathrm{BF}_{4}$ and $\mathbf{d}$ CNT-IPyCl at $\times 100,000$ magnification
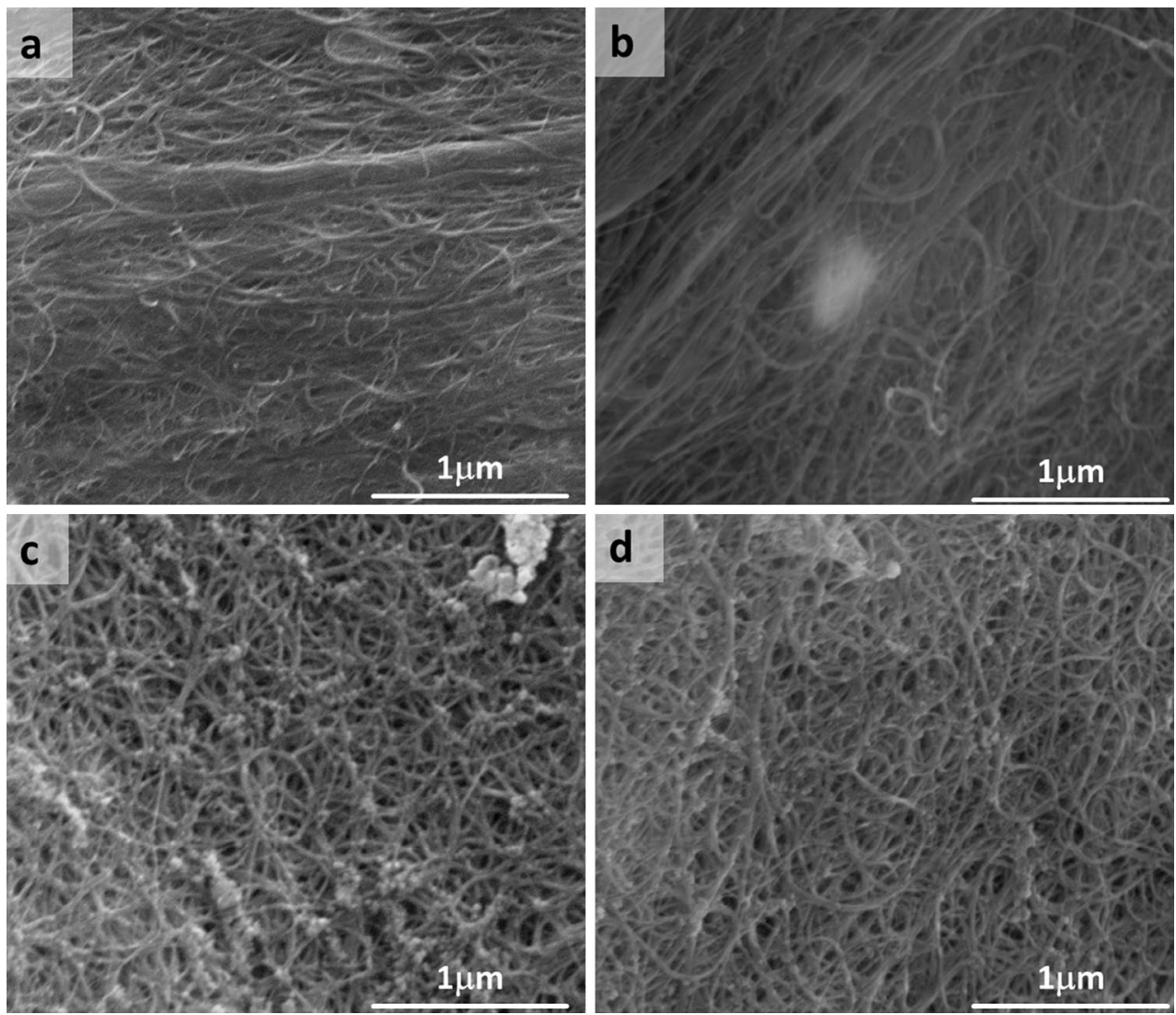

The pristine CNT film (Fig. 5a) was once again characterized by few defects and contaminants (mostly remnants of the catalyst from the synthesis). The constituting CNTs were in very close contact with each other, which translated to a reduced number of voids (and hence the starting material had relatively high electrical conductivity as compared with as will be shown in the further parts of the manuscript). Upon addition of DPIC, the microstructure of the film remained principally unchanged despite the appearance of brighter fragments, similar to mist, which is probably associated with the presence of the DPIC compound on the surface of the film (Fig. 5b). In the case of the films doped with $\mathrm{IPy}_{2} \mathrm{BF}_{4}$ and $\mathrm{IPyCl}$, the structure of which are presented as Fig. 5c, d, the character of the surface is different-small pearls made of the iodine compounds are situated among carbon nanotubes. The SEM structural analysis confirmed that doped films contain the structures other than those typical for the pristine film and, additionally, indicated that the nature of the DPIC compound is different from that of the $\mathrm{IPy}_{2} \mathrm{BF}_{4}$ and $\mathrm{IPyCl}$ compounds, as they look different in the micrographs. After doping by these two iodonium salts, the internal structure of the films changed. Between the bundles, there was created open space supposedly caused by intercalation of these species. As will be shown in the section on electronic and thermal properties of these materials, differences in the internal structures will play an

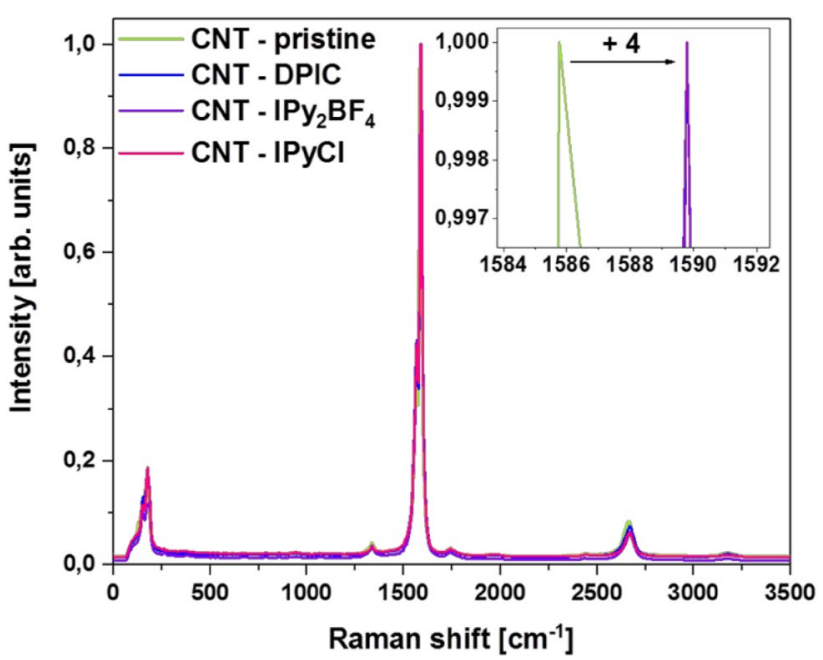

Fig. 6 Raman spectra of undoped and doped SWCNT films

important contribution to the recorded electrical and thermal performance.

We then decided to look into the influence of these compounds on the composition of the nanocarbon material by carrying out Raman spectroscopy (Fig. 6). The $I_{\mathrm{D}} / I_{\mathrm{G}}$ ratio, which is a common way to gauge the level of crystallinity in carbon materials, was calculated and the location of

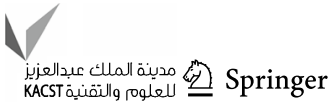


maximum of $\mathrm{G}$ peak was analyzed for every sample. Structural imperfections in the form of $\mathrm{C}_{s p 3}$ groups are detected as so-called D-peak about $1300 \mathrm{~cm}^{-1}$; whereas, the atoms forming $\mathrm{C}_{s p 2}$ lattice give a $\mathrm{G}$ peak about $1600 \mathrm{~cm}^{-1}$. The $I_{\mathrm{D}} / I_{\mathrm{G}}$ ratio of the neat CNT films was found to be as low as 0.035 , which once again validates very low level of disorder in the starting material.

To our delight, we did not observe any change to the value of $I_{\mathrm{D}} / I_{\mathrm{G}}$ ratio in the case of the films doped with iodonium salts. This means that the doping technique elaborated by us has non-destructive character on the structure of CNTs. It should be noted that the maximum of $G$ peak shifted from 1586 to $1589 \mathrm{~cm}^{-1}$, i.e., by $+4 \mathrm{~cm}^{-1}$ (Fig. 6 , inset). This blue shift strongly suggests charge transfer from the nanotube to the dopant (Janas 2018) as expected from the p-doping agents prepared by us for the study.

To get a more detailed insight regarding the chemical composition and type of bonds in the materials, we employed XPS analysis. First, an Auger electron spectrum analysis was conducted to determine the difference (described as $D$ parameter) between the maximum and minimum derivatives of C KLL spectra, in the energy scope of between 1240 and $1190 \mathrm{eV}$. In the case of ideal graphitic structure, its value is 21.4 (Mezzi and Kaciulis 2010; Haerle et al. 2001). Our base
CNT film reached the same value, which again confirms that it is free of defects (Fig. S7).

The analysis of the photoemission peak of $\mathrm{C} 1 s$ (Fig. 7) provided proof that the starting material mainly contains carbon-carbon bonds with $s p^{2}$ hybridization, as indicated by the signal at $248.7 \mathrm{eV}$.

Additionally, there are present minute amounts of functional groups containing oxygen atoms such as $\mathrm{C}-\mathrm{O}$ $(287 \mathrm{eV}), \mathrm{C}=\mathrm{O}$ or $\mathrm{O}-\mathrm{C}=\mathrm{O}(289 \mathrm{eV}), \mathrm{COOH}(290 \mathrm{eV})$ and $\pi-\pi^{*}$ shake-up $(291.4 \mathrm{eV})$. In the case of the samples doped with iodine compounds in the $\mathrm{C} 1 s$ spectrum, an additional signal of the energy of $286 \mathrm{eV}$ is visible, apart from the regular signals visible in the pristine material. It results from the $\mathrm{C}-\mathrm{I}$ and $\mathrm{C}-\mathrm{N}$ type bonds, the peaks of which coincide in this range. It proves that iodine compounds are present in the CNT film and that doping is permanent even under high-vacuum conditions of the XPS.

The doped films were also subject to the study of the photoemission peak of I $3 d$ (Fig. 8).

This step was carried out to learn about the character of the iodine bonds in the material. The results depicted in the Fig. 8 clearly demonstrate that the maxima of peaks in the DPIC compound spectrum (Fig. 8a) are shifted towards higher energies than for the $\mathrm{IPy}_{2} \mathrm{BF}_{4}$ and $\mathrm{IPyCl}$ compounds
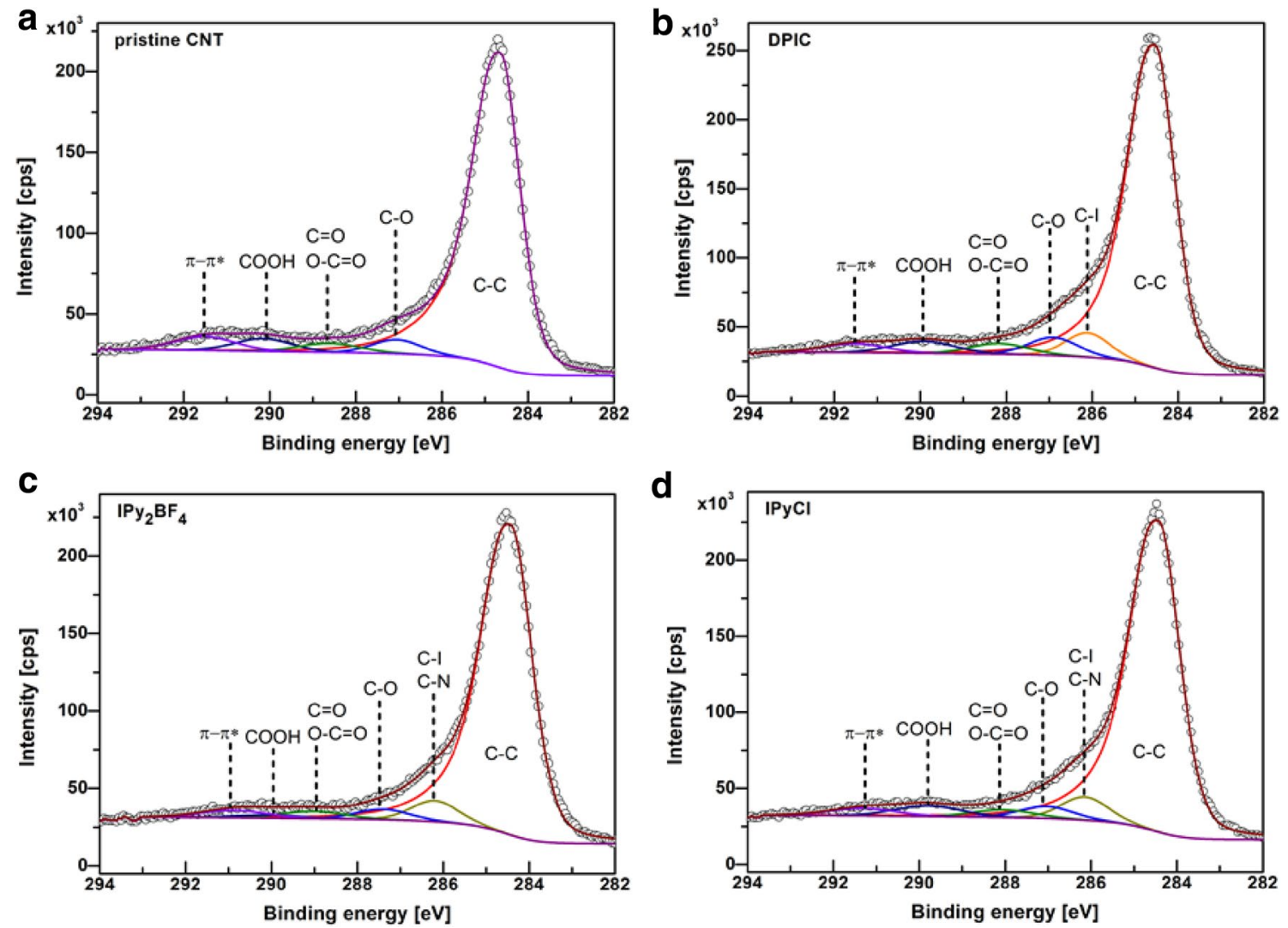

Fig. $7 \mathrm{C} 1 s$ spectra of a pristine CNT film, b CNT-DPIC, $\mathbf{c}$ CNT-IPy ${ }_{2} \mathrm{BF}_{4}$ and $\mathbf{d} \mathrm{CNT}-\mathrm{IPyCl}$ 
a

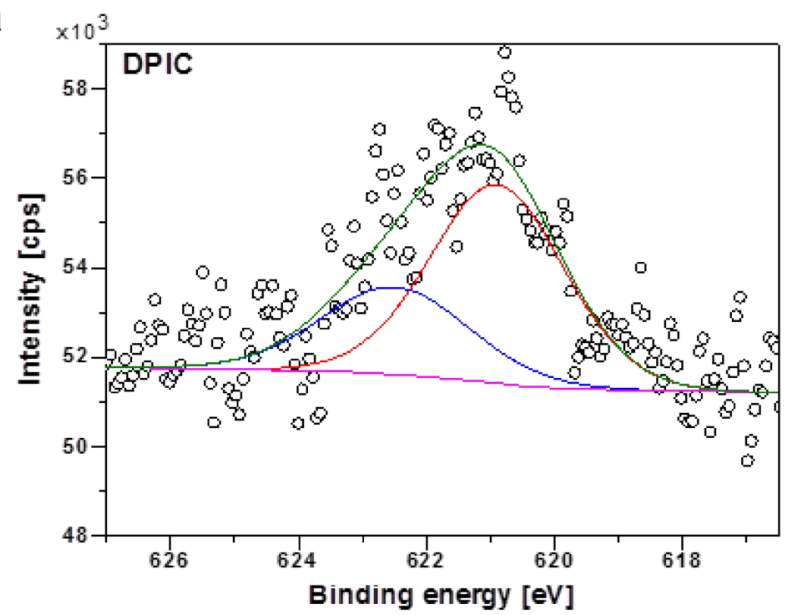

b

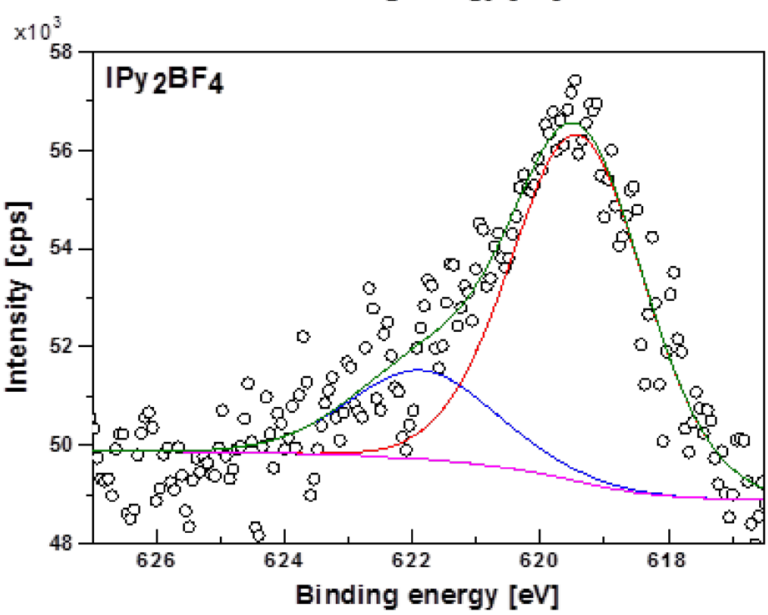

C

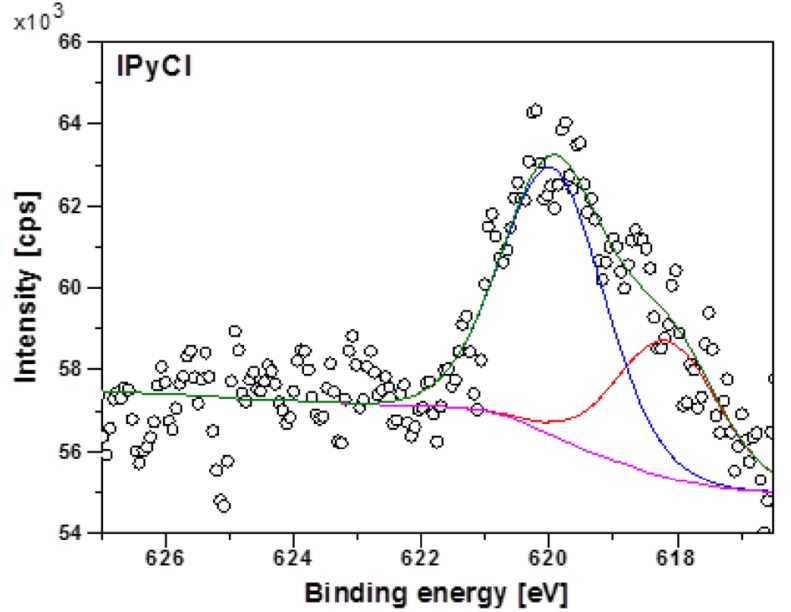

Fig. 8 I $3 d$ spectra of a CNT-DPIC, b CNT-IPy ${ }_{2} \mathrm{BF}_{4}$ and $\mathbf{c}$ CNT$\mathrm{IPyCl}$

(Fig. 8b, c, respectively). This indicates that the electronic configuration in those compounds is different from that of DPIC. Most probably, this is caused by the type of bond between the atoms of iodine and carbon present in the structure of this doping compound. In the case of DPIC, we deal with a covalent bond, so the maximum of the peak can be

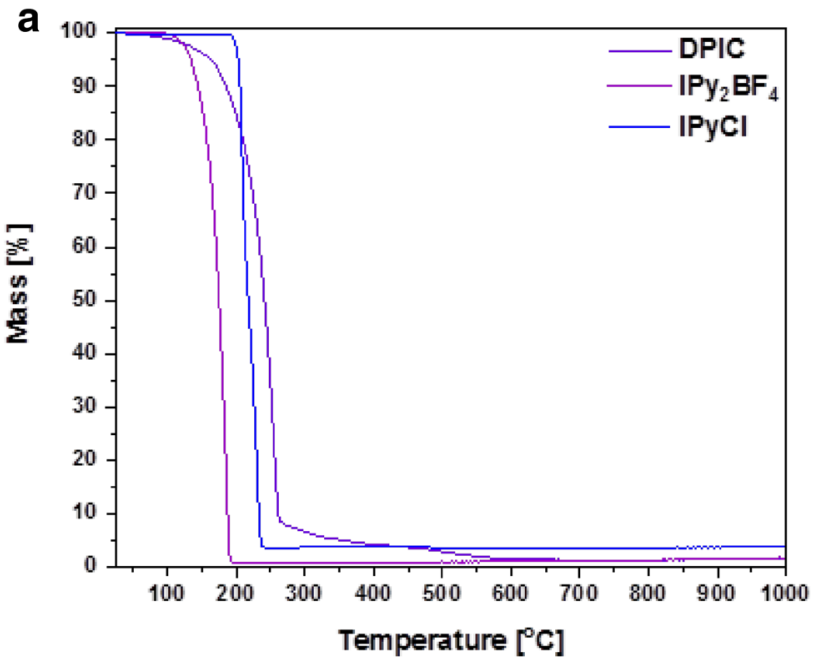

b

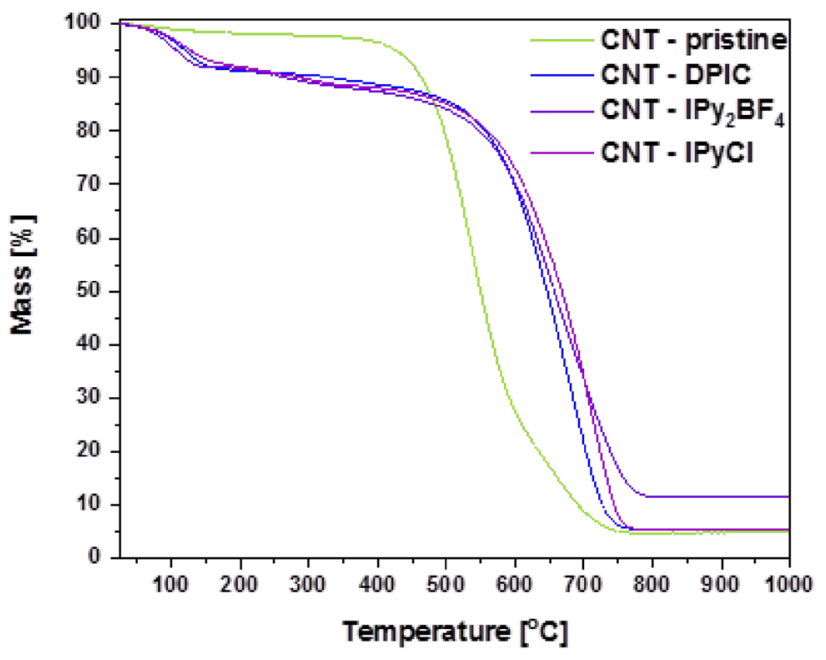

Fig. 9 TGA curves of a iodonium salts and $\mathbf{b}$ the CNT film before and after doping

located at higher energy values than in the case of the coordination bond present in $\mathrm{IPy}_{2} \mathrm{BF}_{4}$ and $\mathrm{IPyCl}$ compounds, which justifies the observed shift. Additionally, it may be observed that in the $\mathrm{IPyCl}$ compound spectrum, the ratio of intensity of the I $3 d$ signal components is opposite to that in the spectra of the other two compounds. That phenomenon probably results from the fact that the compound is more prone to development of coordination bonds with nanotubes than the two previous ones, because it does not have such a strong steric effect, as its structure only contains one aromatic ring, not two. As a consequence, we may suspect that $\mathrm{IPyCl}$ will most readily influence the properties of the CNTs.

Thermogravimetric analysis was used to determine the thermal stability of obtained iodonium salts as such (Fig. 9a) and when incorporated into the CNT networks (Fig. 9b). Each of the thermograms of the iodine compounds displays a single relatively large combustion peak at around $200{ }^{\circ} \mathrm{C}$, 
which corresponds to complete loss of mass on heating. Nevertheless, up to about $100{ }^{\circ} \mathrm{C}$, the thermograms remain relatively flat, so the doping compounds are not prone to desorption in this temperature regime. Thermal analysis was also applied to determine thermal stability of the CNT film and the impact of doping on that (Fig. 9b). The pristine CNT films are characterized by the thermal stability up to $450{ }^{\circ} \mathrm{C}$, and then the oxidation accelerates rapidly as expected for single-walled CNTs. In the case of the films doped with iodine compounds, we observe more complex relation between mass loss and temperature. The first significant change is observable at about $150{ }^{\circ} \mathrm{C}$. That is probably related to the initiation of degradation or desorption of the dopant, which is in accordance with the result obtained during thermogravimetric analysis of pure iodine substances.

The XRD analysis indicated that the applied doping with iodonium salts does not affect the crystallographic structure of CNTs. The doped CNT films do not demonstrate additional reflexes that would illustrate such change (Fig. S8).

The start of second notable change in mass of CNT samples can be ascribed to about $550{ }^{\circ} \mathrm{C}$, which demonstrates that doping increases the thermal stability of films by about $100{ }^{\circ} \mathrm{C}$ (pristine CNT film started the decomposition at circa $450{ }^{\circ} \mathrm{C}$ ). This can be explained by the observed modification of the microstructure as visualized earlier by electron microscopy. Our previous studies show that the packing degree can have a strong effect on the shape of the thermograms (Janas et al. 2013a). From the chemical point of view, it is important to consider that halogen-bearing species can purify the material from low-molecular weight contamination, which effectively increases its thermal stability (Janas et al. 2014) and that is what we witnessed.

To demonstrate that the change of microstructure and electronic structure upon doping gives rise to tangible

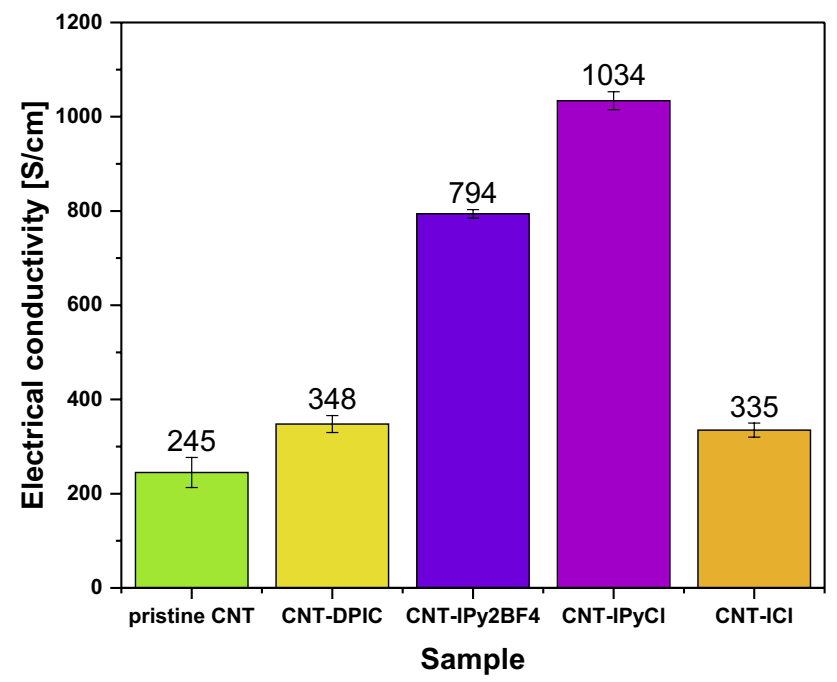

Fig. 10 Electrical conductivity of measured CNT films enhancement of the properties of the CNT films, we evaluated their electrical and thermal conductivity. The measurements on the electrical front showed that the CNT film doped with a DPCI compound has gained just slight improvement of electrical conductivity $(348 \pm 18 \mathrm{~S} / \mathrm{cm}$ vs. $245 \pm 32 \mathrm{~S} / \mathrm{cm}$ for the untreated film, Fig. 10).

The boost was on the same level as when iodine monochloride (reported previously as strong doping agent of nanocarbon (Janas et al. 2014, 2017a) was employed as reference, which improved electrical conductivity of the CNT film up to $335 \pm 15 \mathrm{~S} / \mathrm{cm}$. The $\mathrm{IPy}_{2} \mathrm{BF}_{4}$ and $\mathrm{IPyCl}$ compounds demonstrated much greater improvement of electrical conductivity, i.e., by $224 \%$ and $322 \%$ in comparison with the starting material (reaching $794 \pm 9 \mathrm{~S} / \mathrm{cm}$ and $1034 \pm 19 \mathrm{~S} / \mathrm{cm}$, respectively). Various degree of enhancement of electrical conductivity of CNT films stems from the differences between their structures and type of $\mathrm{C}-\mathrm{I}$ bonding. As indicated by XPS analysis, the $\mathrm{IPy}_{2} \mathrm{BF}_{4}$ and $\mathrm{IPyCl}$ compounds have similar electron structure with labile iodine atom, and so adding them to SWCNTs resulted in a significant improvement of electrical conductivity. It was more profound in the case if $\mathrm{IPyCl}$ because there was no steric hindrance and the $\mathrm{I}^{+}$cation was more likely to interact with the carbon lattice because of higher "unsaturation" of its electronic shell. In the case of DPCI and iodine chloride, their electron structure is also similar. Iodine atoms are bound with strong covalent bonds, so the impact on the improvement of electrical conductivity is not as strong as in the case of $\mathrm{IPy}_{2} \mathrm{BF}_{4}$ and $\mathrm{IPyCl}$, wherein coordination bonds are present. Furthermore, as can be seen in SEM micrographs, the microstructural changes that appeared after doping with $\mathrm{IPy}_{2} \mathrm{BF}_{4}$ and $\mathrm{IPyCl}$ are analogous. Based on the change in the microstructure, we suspect that these chemical species can more readily cause intercalation of the

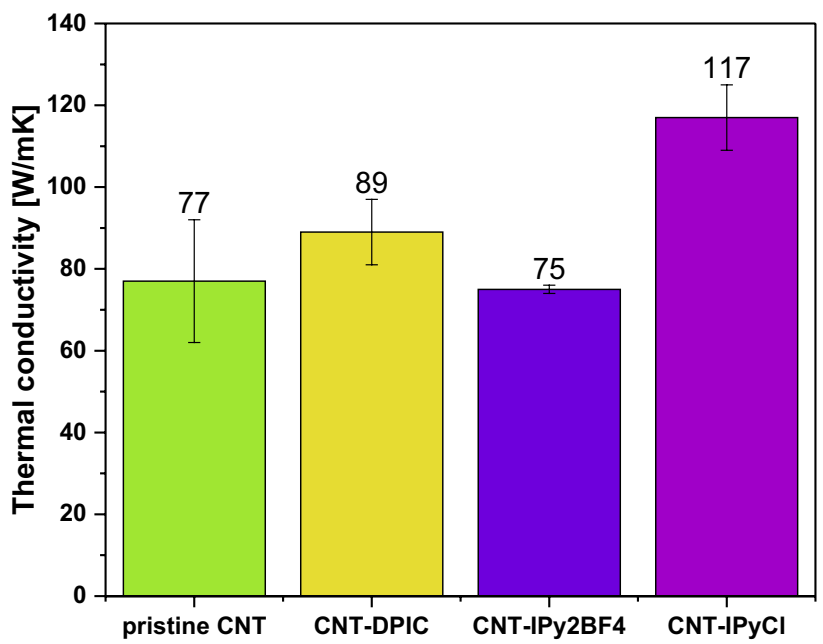

Fig. 11 Thermal conductivity of measured CNT films 
CNT network with iodine atoms, which is responsible for the higher increase in electrical conductivity.

Finally, the steady-state method with infrared thermography was used to measure thermal conductivity of the CNT films before and after doping (Fig. 11). The thermal conductivity of pristine film was found to be about $77 \pm 15 \mathrm{~W} / \mathrm{mK}$. Our result match the thermal conductivity of the CNT systems similar to ours, also created from isotropically distributed single-walled carbon nanotubes, which were measured by the same method (Feng et al. 2018; Wang et al. 2008; Nasibulin et al. 2011). What is more, the value of thermal conductivity of the films doped with compounds of DPCI and $\mathrm{IPy}_{2} \mathrm{BF}_{4}$ stayed at the same level as measured for the pristine film $(89 \pm 9 \mathrm{~W} / \mathrm{mK}$ and $75 \pm 1 \mathrm{~W} / \mathrm{mK}$, respectively). On the other hand, in the case of IPyCl doping, we observed an increase in the value of thermal conductivity by over $50 \%$ reaching $117 \pm 8 \mathrm{~W} / \mathrm{mK}$. As discussed before, IPyCl geometry and electronic structure are somewhat unique and can readily donate iodine atoms to the CNT ensemble because of the type of $\mathrm{C}-\mathrm{I}$ bonding and lack of steric hindrance. Previous results indicate that doping of SWCNTs with iodine can markedly increase thermal conductivity of CNT networks (Behabtu et al. 2013; Mayhew and Prakash 2014). It appears that using our compounds can also be beneficial in this regard but the effect is most profound in the case of IPyCl doping because of the aforementioned justification why it is most compatible with nanocarbon due to its electronic and structural features.

\section{Conclusions}

In summary, we have developed an efficient and convenient method for the modification of properties of CNT films by means of doping with iodonium salts. Our methodology provides a novel approach to obtain iodine-doped CNTs, which hitherto are limited by previous conventional methods.

The highest increase in electrical and thermal conductivity was observed for the SWCNTs doped with IPyCl, which enhanced them by over $4 \times$ and $1.5 \times$ times, respectively, as compared with un-doped SMCNT films. Both the electronic structure and open geometry of a IPyCl molecule make doping with that compound most effective in terms of improvement of the transport properties of the explored nanocarbon material. In the case of other employed iodine compounds $\left(\mathrm{IPy}_{2} \mathrm{BF}_{4}\right.$ and DPIC), we also noted improvements to the properties of the CNT films, but they were much less notable. This was caused by the presence of a stronger $\mathrm{C}-\mathrm{I}$ covalent bond and/or the steric hindrance, which limited the lability of the iodine atoms which can cause intercalation of the CNT films.

Based on these results, we can state that out of the iodine compounds which we synthesized, $\mathrm{IPyCl}$ is most promising to effectively improve the overall performance of the CNT network. We believe that synthesis of IPyCl analogues could lead to similar favorable improvements, and so the direction proposed by us to the nanocarbon society could be an attractive alternative as compared with the use of iodine vapors or hazardous iodine chemicals to reach the same goal. From the practical point of view, incorporation of stable iodine species into the CNT network to tune the transport performance could be an important route towards design of highperformance components for microelectronics based on a wider spectrum of nanocarbon materials.

Acknowledgements B.K. and D.J. thank the National Centre for Research and Development, Poland (under the Leader program, Grant Agreement LIDER/0001/L-8/16/NCBR/2017). P.S.W. thanks the National Science Centre for the financial support within the frames of the Preludium program (Grant Agreement 2018/29/N/ST8/02356). L.P. and K.Z.W. thank the Silesian University of Technology for the financial support (Grant Agreement BKM/534/RCh-2/0044).

Author contributions The manuscript was written through contributions of all authors. All authors have given approval to the final version of the manuscript.

\section{Compliance with ethical standards}

Conflict of interest The authors have to declare no conflict of interest.

Open Access This article is distributed under the terms of the Creative Commons Attribution 4.0 International License (http://creativeco mmons.org/licenses/by/4.0/), which permits unrestricted use, distribution, and reproduction in any medium, provided you give appropriate credit to the original author(s) and the source, provide a link to the Creative Commons license, and indicate if changes were made.

\section{References}

Barluenga J, Gonzalez JM, Garcia-Martin MA et al (1993) Acid-mediated reaction of bis(pyridine)iodonium(I) tetrafluoroborate with aromatic compounds. A selective and general iodination method. J Org Chem 58:2058-2060. https://doi.org/10.1021/jo00060a020

Barluenga J, Álvarez-Gutiérrez JM, Ballesteros A, González JM (2007) Directortho iodination of $\beta$ - and $\gamma$-aryl alkylamine derivatives. Angew Chemie Int Ed 46:1281-1283. https://doi.org/10.1002/ anie. 200603631

Behabtu N, Young CC, Tsentalovich DE et al (2013) Strong, light, multifunctional fibers of carbon nanotubes with ultrahigh conductivity. Science (80-) 339:182-186. https://doi.org/10.1126/ science. 1228061

Berber S, Kwon Y-K, Tomanek D (2000) Unusually high thermal conductivity of carbon nanotubes. Phys Rev Lett 84:4613-4616. https ://doi.org/10.1103/PhysRevLett.84.4613

Blackburn JL, Ferguson AJ, Cho C, Grunlan JC (2018) Carbon-nanotube-based thermoelectric materials and devices. Adv Mater 30:1704386. https://doi.org/10.1002/adma.201704386

Borzenko A, Rotta-Loria NL, MacQueen PM et al (2015) Nickelcatalyzed monoarylation of ammonia. Angew Chemie Int Ed 54:3773-3777. https://doi.org/10.1002/anie.201410875

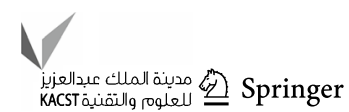


Brady GJ, Way AJ, Safron NS et al (2016) Quasi-ballistic carbon nanotube array transistors with current density exceeding $\mathrm{Si}$ and GaAs. Sci Adv 2:e1601240. https://doi.org/10.1126/sciadv.1601240

Chen J-H, Jang C, Xiao S et al (2008) Intrinsic and extrinsic performance limits of graphene devices on $\mathrm{SiO}_{2}$. Nat Nanotechnol 3:206-209. https://doi.org/10.1038/nnano.2008.58

Dettlaff-Weglikowska U, Skákalová V, Graupner R et al (2005) Effect of $\mathrm{SOCl}_{2}$ treatment on electrical and mechanical properties of single-wall carbon nanotube networks. J Am Chem Soc 127:51255131. https://doi.org/10.1021/ja046685a

Espuña G, Arsequell G, Valencia G et al (2004) Regioselective postsynthetic modification of phenylalanine side chains of peptides leading to uncommonortho-iodinated analogues. Angew Chemie Int Ed 43:325-329. https://doi.org/10.1002/anie.200352464

Fanchini G, Unalan HE, Chhowalla M (2007) Modification of transparent and conducting single wall carbon nanotube thin films via bromine functionalization. Appl Phys Lett 90:092114. https://doi. org/10.1063/1.2709903

Feng Y, Inoue T, Watanabe M et al (2018) Measurement of in-plane sheet thermal conductance of single-walled carbon nanotube thin films by steady-state infrared thermography. Jpn J Appl Phys 57:075101. https://doi.org/10.7567/JJAP.57.075101

Friedman JS, Girdhar A, Gelfand RM et al (2017) Cascaded spintronic logic with low-dimensional carbon. Nat Commun 8:15635. https ://doi.org/10.1038/ncomms15635

Fujii M, Zhang X, Xie H et al (2005) Measuring the thermal conductivity of a single carbon nanotube. Phys Rev Lett 95:8-11. https:// doi.org/10.1103/PhysRevLett.95.065502

Fukumaru T, Fujigaya T, Nakashima N (2015) Development of n-type cobaltocene-encapsulated carbon nanotubes with remarkable thermoelectric property. Sci Rep 5:7951. https://doi.org/10.1038/srep0 7951

Gao C, Guo Z, Liu J-H, Huang X-J (2012) The new age of carbon nanotubes: an updated review of functionalized carbon nanotubes in electrochemical sensors. Nanoscale 4:1948. https://doi. org/10.1039/c2nr11757f

Gonnet P, Liang Z, Choi ES et al (2006) Thermal conductivity of magnetically aligned carbon nanotube buckypapers and nanocomposites. Curr Appl Phys 6:119-122. https://doi.org/10.1016/J. CAP.2005.01.053

Haerle R, Riedo E, Pasquarello A, Baldereschi A (2001) $\mathrm{sp}^{2} / \mathrm{sp}^{3}$ hybridization ratio in amorphous carbon from $\mathrm{C} 1 \mathrm{~s}$ core-level shifts: X-ray photoelectron spectroscopy and first-principles calculation. Phys Rev B 65:045101. https://doi.org/10.1103/PhysR evB.65.045101

Hamri S, Rodríguez J, Basset J et al (2012) A convenient iodination of indoles and derivatives. Tetrahedron 68:6269-6275. https://doi. org/10.1016/J.TET.2012.05.053

He M, Swager TM (2016) Covalent functionalization of carbon nanomaterials with iodonium salts. Chem Mater 28:8542-8549. https ://doi.org/10.1021/acs.chemmater.6b03078

Hong S, Myung S (2007) A flexible approach to mobility. Nat Nanotechnol 2:207-208. https://doi.org/10.1038/nnano.2007.89

Iijima S (1991) Helical microtubules of graphitic carbon. Nature 354:56-58. https://doi.org/10.1038/354056a0

Iskra J, Murphree SS (2017) Rapid aerobic iodination of arenes mediated by hypervalent iodine in fluorinated solvents. Tetrahedron Lett 58:645-648. https://doi.org/10.1016/J.TETLET.2017.01.003

Janas D (2018) Powerful doping of chirality-sorted carbon nanotube films. Vacuum 149:48-52. https://doi.org/10.1016/J.VACUU M.2017.12.013

Janas D, Stando G (2017) Unexpectedly strong hydrophilic character of free-standing thin films from carbon nanotubes. Sci Rep 7:12274. https://doi.org/10.1038/s41598-017-12443-y
Janas D, Cabrero-Vilatela A, Bulmer J et al (2013a) Carbon nanotube wires for high-temperature performance. Carbon N Y 64:305-314. https://doi.org/10.1016/J.CARBON.2013.07.067

Janas D, Vilatela AC, Koziol KKK (2013b) Performance of carbon nanotube wires in extreme conditions. Carbon N Y 62:438-446. https://doi.org/10.1016/J.CARBON.2013.06.029

Janas D, Boncel S, Koziol KKK (2014) Electrothermal halogenation of carbon nanotube films. Carbon N Y 73:259-266. https://doi. org/10.1016/J.CARBON.2014.02.062

Janas D, Milowska KZ, Bristowe PD, Koziol KKK (2017a) Improving the electrical properties of carbon nanotubes with interhalogen compounds. Nanoscale 9:3212-3221. https://doi.org/10.1039/ C7NR00224F

Janas D, Rdest M, Koziol KKK (2017b) Free-standing films from chirality-controlled carbon nanotubes. Mater Des 121:119-125. https://doi.org/10.1016/J.MATDES.2017.02.062

Javey A, Qi P, Wang Q, Dai H (2004) Ten- to 50-nm-long quasi-ballistic carbon nanotube devices obtained without complex lithography. Proc Natl Acad Sci USA 101:13408-13410. https://doi. org/10.1073/pnas.0404450101

Kumanek B, Janas D (2019) Thermal conductivity of carbon nanotube networks—review. J Mater Sci 54:7397-7427. https://doi. org/10.1007/s10853-019-03368-0

Landi BJ, Ganter MJ, Cress CD et al (2009) Carbon nanotubes for lithium ion batteries. Energy Environ Sci 2:638. https://doi. org/10.1039/b904116h

Lee RS, Kim HJ, Fischer JE et al (1997) Conductivity enhancement in single-walled carbon nanotube bundles doped with $\mathrm{K}$ and $\mathrm{Br}$. Nature 388:255-257. https://doi.org/10.1038/40822

Liu P, Fan Z, Mikhalchan A et al (2016a) Continuous carbon nanotubebased fibers and films for applications requiring enhanced heat dissipation. ACS Appl Mater Interfaces 8:17461-17471. https:// doi.org/10.1021/acsami.6b04114

Liu Y, Shen Y, Sun L et al (2016b) Elemental superdoping of graphene and carbon nanotubes. Nat Commun 7:10921. https://doi. org/10.1038/ncomms10921

Liu Y, Huang D, Huang J, Maruoka K (2017) Hypervalent iodine mediated chemoselective iodination of alkynes. J Org Chem 82:1186511871. https://doi.org/10.1021/acs.joc.7b01555

Mayhew E, Prakash V (2014) Thermal conductivity of high performance carbon nanotube yarn-like fibers. J Appl Phys 115:174306. https://doi.org/10.1063/1.4874737

McEuen PL, Fuhrer MS, Park Hongkun (2002) Single-walled carbon nanotube electronics. IEEE Trans Nanotechnol 1:78-85. https:// doi.org/10.1109/TNANO.2002.1005429

Merritt E, Olofsson B (2009) Diaryliodonium salts: a journey from obscurity to fame. Angew Chemie Int Ed 48:9052-9070. https:// doi.org/10.1002/anie.200904689

Mezzi A, Kaciulis S (2010) Surface investigation of carbon films: from diamond to graphite. Surf Interface Anal 42:1082-1084. https:// doi.org/10.1002/sia.3348

Nasibulin AG, Kaskela A, Mustonen K et al (2011) Multifunctional free-standing single-walled carbon nanotube films. ACS Nano 5:3214-3221. https://doi.org/10.1021/nn200338r

Przypis L, Walczak KZ (2019) Copper(II)-catalyzed iodinations of carbazoles: access to functionalized carbazoles. J Org Chem 84:2287-2296. https://doi.org/10.1021/acs.joc.8b02821

Qiu L, Zou H, Zhu N et al (2018) Iodine nanoparticle-enhancing electrical and thermal transport for carbon nanotube fibers. Appl Therm Eng 141:913-920. https://doi.org/10.1016/J.APPLT HERMALENG.2018.06.049

Racys DT, Sharif SAI, Pimlott SL, Sutherland A (2016) Silver(I)-catalyzed iodination of arenes: tuning the lewis acidity of $N$-iodosuccinimide activation. J Org Chem 81:772-780. https://doi. org/10.1021/acs.joc.5b02761 
Tonkikh AA, Tsebro VI, Obraztsova EA et al (2015) Metallization of single-wall carbon nanotube thin films induced by gas phase iodination. Carbon N Y 94:768-774. https://doi.org/10.1016/J. CARBON.2015.07.062

Wang J (2005) Carbon-nanotube based electrochemical biosensors: a review. Electroanalysis 17:7-14. https://doi.org/10.1002/ elan.200403113

Wang D, Song P, Liu C et al (2008) Highly oriented carbon nanotube papers made of aligned carbon nanotubes. Nanotechnology 19:075609. https://doi.org/10.1088/0957-4484/19/7/075609

Wu Z, Chen Z, Du X et al (2004) Transparent, conductive carbon nanotube films. Science 305:1273-1276. https://doi.org/10.1126/scien ce. 1101243

Yoshimura A, Zhdankin VV (2016) Advances in synthetic applications of hypervalent iodine compounds. Chem Rev 116:3328-3435. https://doi.org/10.1021/acs.chemrev.5b00547

Zhan Y, Zhang B, Cao L et al (2015) Iodine doped graphene as anode material for lithium ion battery. Carbon N Y 94:1-8. https://doi. org/10.1016/J.CARBON.2015.06.039
Zhang L, Zhang G, Liu C, Fan S (2012) High-density carbon nanotube buckypapers with superior transport and mechanical properties. Nano Lett 12:4848-4852. https://doi.org/10.1021/nl3023274

Zhao Y, Wei J, Vajtai R et al (2011) Iodine doped carbon nanotube cables exceeding specific electrical conductivity of metals. Sci Rep 1:83. https://doi.org/10.1038/srep00083

Zhou X, Park J-Y, Huang S et al (2005) Band structure, phonon scattering, and the performance limit of single-walled carbon nanotube transistors. Phys Rev Lett 95:146805. https://doi.org/10.1103/ PhysRevLett.95.146805

Publisher's Note Springer Nature remains neutral with regard to jurisdictional claims in published maps and institutional affiliations. 